\section{COVID-19: Introducing Low Dose Radiation as an Effective Treatment for Pneumonia that Shouldn't Induce Selective Pressure and New Mutations}

\author{
Mehdizadeh A. R. ${ }^{1 \oplus}$, Bevelacqua J. J.2, Mortazavi S. \\ A. R. ${ }^{3}$, Mortazavi S. M. J. ${ }^{1 * \odot}$
}

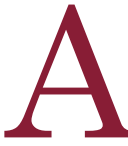

s of May 31, 2020, severe acute respiratory syndrome coronavirus 2 (SARS-CoV-2) has infected more than 6.1 million people worldwide, and more than 370,000 have now died with the coronavirus. The novel coronavirus disease (COVID-19) can be transmitted by asymptomatic patients that makes preventing the spread of this disease much more challenging [1]. Currently, there are no known available effective antiviral drugs for treatment of COVID-19 patients. A National Institutes of Health $(\mathrm{NIH})$ panel has recently released new guidelines on treating patients with COVID-19. The panel did not recommend any drug for pre- or post-exposure prophylaxis outside of clinical trials. Moreover, as no drug has been proven to be safe and effective so far, the panel does not make a recommendation for specific antiviral or immunomodulatory treatments [2]. The US CDC (Centers for Disease Control and Prevention) believes that a COVID-19 outbreak could persist for a long time in some communities [3]. Considering the rapid doubling rate of COVID-19 cases, a serious public health problem can be expected, even in well-developed countries with advanced medical and healthcare systems. A large case series of 72,314 infected individuals $(62 \%$ of diagnoses based on positive viral nucleic acid test results in throat swab samples) in China showed severe disease in $14 \%$ of the infected persons and a case-fatality rate (CFR) of $2.3 \%$. However, as of March 17, 2020, the CFR for COVID-19 in Italy was 7.2\%, which is much higher than that observed in other countries [4].

During the first half of the $20^{\text {th }}$ century, X-ray therapy successfully treated pneumonia [5]. As noted in a review by Calabrese and Dhawan [5], fifteen studies reported that approximately 700 cases of various types of pneumonia were effectively treated by low doses of X-rays. These treatments led to disease resolution, based on clinical symptoms, objective disease biomarkers, and mortality incidence. A team of Iranian and American scientists introduced the idea of using low dose radiation therapy (LDRT) for COVID-19 related pneumonia for the first time [6].
${ }^{1} \mathrm{PhD}$, Department of Medical Physics, School of Medicine, Shiraz University of Medical Sciences, Shiraz, Iran ${ }^{2} \mathrm{PhD}$, Bevelacqua

Resources, Richland, Washington 99352. United States

${ }^{3} \mathrm{MD}$, School of Medicine, Shiraz University of Medical Sciences,

Shiraz, Iran

*Corresponding author: S. M. J. Mortazavi Department of Medical Physics, School of Medicine, Shiraz University of Medical Sciences,

Shiraz, Iran

E-mail: mortazavismj@ gmail.com

Received: 3 May 2020 Accepted: 10 May 2020 
These investigators proposed a modified effective method for managing pneumonia in COVID-19 patients with the minimal negative effects. In their proposed method, patients should receive a single dose of either 100, 180 or 250 mGy X-ray after a pre-exposure to a conditioning dose. However, it is an open issue if lung irradiation has all the immune system stimulation advantages of a whole-body irradiation. These investigators also addressed the advantages of LDR over treatment methods such as those use antiviral drugs. They raised the concerns over unintentional shift of the SARS-CoV-2 to more resistant types. Now, we know that when the treatment is not effective and viral genome replicates, selective pressure leads the virus to rapid adaptation toward resistance "An increasing number of viral infections that impair host health are treated using antiviral drugs, typically targeting mechanisms of viral replication. If the treatment is robust and viral fitness is impaired sufficiently, no viral genomes will be successfully replicated, but if treatment is not as effective and some genomes replicate, selective pressure may result in rapid adaptation toward resistance" [7]. Ghadimi-Moghadam et al. [6] noted that LDR not only has the capacity of modulating excessive inflammatory responses, but regulates the lymphocyte counts, and controls bacterial co-infections in patients with COVID-19. LDR mitigates inflammation and facilitates healing via the polarization of macrophages to an anti-inflammatory or M2 phenotype [8]. Moreover, inducing apoptosis in radiosensitive neutrophils and activated T cells, and inhibiting endothelial/ leukocyte interaction resulting in reduced trafficking of infiltrating immune cells are additional proposed mechanisms by which LDR mitigates inflammation and facilitates healing.

Given this consideration, a single dose of either 100, 180 or $250 \mathrm{mGy}$ of X-rays cannot exert a significant selective pressure on SARS-CoV-2, and hence does not lead the virus to "accelerated evolution" through mutations. LDR uses its capacity of modulating excessive inflammatory responses, regulates lymphocyte counts, and controls bacterial co-infections in patients with COVID-19.

Just a short time after this publication, the potential of low dose radiation therapy as a treatment for COVID-19 pneumonia received attention globally. Canadian scientists from the Universities of Calgary and Alberta published a letter and suggested that despite some obstacles (e.g., existing strain on radiotherapy resources and separating COVID-19 patients and cancer patients), conducting clinical trials to further investigate the efficacy of whole lung low dose radiation therapy (LDRT) would present a very low risk treatment for COVID-19 pneumonia patients. They stated that LDRT has the potential to reduce mortality and alleviate COVID-19 related strains on healthcare systems [9]. Subsequently, Spanish [10-11], French [12-13], American [14], German [15] and Indian [16] scientists also addressed the importance of LDRT in these treatments. Now a paper published in the Lancet [17] raises the question whether anticancer radiation therapy may have protective role against the cytokine storm caused by SARS-CoV-2? Moreover, a literature search shows that groups of scientists from different parts of the world have also planned for conducting relevant experiments.

Moreover, new reports show that COVID-19 cannot be considered a standard variety, contagious and lethal respiratory disease with no vaccine and no treatment, but also a real threat to kidneys, heart, intestines, liver and brain. Storms of blood clots have been reported in some hospitalized patients with COVID-19 [18]. Studies show that clots occur in 20-30\% of terminally ill COVID-19 patients [19-20]. Given this consideration, a main question is if LDRT can reduce the occurrence of blood clots? Considering the point that oxidative stress plays an important role in clotting of blood through mechanisms such as enhanced generation of isoprostanes [21], it can be hypothesized that LDRT would decrease or block the clotting of blood through reducing oxidative stress. This mechanism would require low-dose radiation for the whole body, not just to the lungs. 
Well-designed studies are needed to evaluate the validity of this hypothesis. There are additional open issues that merit future investigation. In particular, physicians and scientists remain unsure regarding the specific effects of LDR on inflammation and how it is mitigated by various irradiation-based treatment modes. In spite of these uncertainties, low dose irradiation provides a possible beneficial treatment protocol to alleviate COVID-19 related strains on healthcare systems.

\section{Conflict of Interest}

None

\section{References}

1. Yao HP, Lu X, Chen Q, Xu K, Chen Y, Cheng L, Liu F, Wu Z, Wu H, Jin C, Zheng M. Patient-derived mutations impact pathogenicity of SARS-CoV-2. medRxiv. 2020. doi: 10.1101/2020.04.14.20060160.

2. Young K. NIH Publishes COVID-19 Treatment Guidelines. Medical News I PHYSICIAN'S FIRST WATCH; David G. Fairchild, and Lorenzo Di Francesco, editors. NIH; 2020.

3. Desai AN, Patel P. Stopping the spread of COVID-19. Jama. 2020;323(15):1516. doi: 10.1001/jama.2020.4269.

4. Onder G, Rezza G, Brusaferro S. Case-fatality rate and characteristics of patients dying in relation to COVID-19 in Italy. Jama. 2020.

5. Calabrese EJ, Dhawan G. How radiotherapy was historically used to treat pneumonia: could it be useful today? Yale J Biol Med. 2013;86(4):555-70. PubMed PMID: 24348219. PubMed PMCID: PMC3848110

6. Ghadimi-Moghadam A, Haghani M, Bevelacqua JJ, Jafarzadeh A, et al. COVID-19 tragic pandemic: concerns over unintentional "directed accelerated evolution" of novel Coronavirus (SARS-CoV-2) and introducing a modified treatment method for ARDS. J Biomed Phys Eng. 2020;10(2):241-6. doi: 10.31661/jbpe.v0i0.2003-1085. PubMed PMID: 32337192. PubMed PMCID: PMC7166223.

7. Irwin KK, Renzette N, Kowalik TF, Jensen JD. Antiviral drug resistance as an adaptive process. Virus Evol. 2016;2(1):vew014. doi: 10.1093/ve/vew014. PMID: 28694997. PMCID: PMC5499642.

8. Porta C, Riboldi E, Ippolito A, Sica A. Molecular and epigenetic basis of macrophage polarized activation. Seminars in immunology. 2015;27(4):237-48. doi: 10.1016/j.smim.2015.10.003.

9. Kirkby C, Mackenzie M. Is low dose radiation therapy a potential treatment for COVID-19 pneumonia? Radiotherapy and Oncology. 2020. doi: 10.1016/j.radonc.2020.04.004. PubMed PMID:32342871. PubMed PMCID: PMC7194710.

10. Lara PC, Burgos J, Macias D. Low dose lung radiotherapy for COVID-19 pneumonia. The rationale for a cost-effective anti-inflammatory treatment. Clinical and Translational Radiation Oncology. 2020;23:27-9. doi: 10.1016/j. ctro.2020.04.006.

11. Montero A, Arenas M, Algara M. Low-dose radiation therapy: could it be a game-changer for COVID-19? Clinical \& Translational Oncology. 2020:1. doi: 10.1007/s12094-020-02401-y.

12. Cosset JM, Deutsch É, Bazire L, Mazeron JJ, Chargari C. Irradiation pulmonaire ā faible dose pour l'orage de cytokines du covid-19: pourquoi pas? Cancer Radiother. 2020. doi: 10.1016/j.canrad.2020.04.003. PubMed PMID: 32389579. PubMed PMCID: PMC7252150.

13. Meziani L, Robert C, Mordant $P$, Deutsch E. Low doses of radiation therapy increase the immunosuppressive profile of lung macrophages via IL-10 production and IFN $\gamma / \mathrm{IL}-6$ suppression: a therapeutic strategy to counteract lung inflammation? bioRxiv. 2020. doi: 10.1101/2020.05.11.0776511.

14. Dhawan G, Kapoor R, Dhawan R, Singh R, Monga B, Giordano J, Calabrese EJ. Low dose radiation therapy as a potential life saving treatment for COVID-19-induced acute respiratory distress syndrome (ARDS). Radiotherapy and Oncology. 2020. doi: 10.1016/j.radonc.2020.05.002.

15. Rödel F, Arenas M, Ott OJ, Fournier C, Georgakilas AG, Tapio S, Trott KR, Gaipl US. Low-dose radiation therapy for COVID-19 pneumopathy: what is the evidence?. Strahlentherapie und Onkologie. 2020:1-4. doi: 10.1007/ s00066-020-01635-7.

16. Venkatraman P, Sahay JJ, Maidili T, Rajan R, Pooja S. Breakthrough of COVID-19 using radiotherapy treatment modalities. Radiotherapy and Oncology. 2020;148:225-6. doi: 10.1016/j.radonc.2020.04.024. 
17. Poortmans PM, Guarneri V, Cardoso M-J. Cancer and COVID-19: what do we really know? The Lancet. 2020. doi.org/10.1016/S0140-6736(20)31240-X.

18. Willyard C. Coronavirus blood-clot mystery intensifies. Nature. 2020.

19. Klok FA, Kruip MJH, Van Der Meer NJM, Arbous MS, Gommers D, Kant KM, Kaptein FHJ, Van Paassen J, Stals MAM, Huisman MV, Endeman $\mathrm{H}$. Confirmation of the high cumulative incidence of thrombotic complications in critically ill ICU patients with COVID-19: An updated analysis. Thromb Res. 2020. doi: 10.1016/j. thromres.2020.04.041.

20. Poissy J, Goutay J, Caplan M, Parmentier E, Duburcq T, Lassalle F, Jeanpierre E, Rauch A, Labreuche J, Susen S. Pulmonary embolism in COVID-19 patients: awareness of an increased prevalence. Circulation. 2020. doi: 10.1161/CIRCULATIONAHA.120.047430.

21. Violi F, Pignatelli P. Platelet oxidative stress and thrombosis. Thrombosis research. 2012;129(3):378-81. doi: 10.1016/j.thromres.2011.12.002. 